\title{
Cell Death and Ageing - A Question of Cell Type
}

\author{
Pidder Jansen-Dürr \\ Institute for Biomedical Ageing Research, Rennweg 10, 6020 Innsbruck, Austria
}

Received December 19, 2001; Revised February 6, 2002; Accepted February 11, 2002; Published April 9, 2002

\begin{abstract}
Replicative senescence of human cells in primary culture is a widely accepted model for studying the molecular mechanisms of human ageing. The standard model used for studying human ageing consists of fibroblasts explanted from the skin and grown into in vitro senescence. From this model, we have learned much about molecular mechanisms underlying the human ageing process; however, the model presents clear limitations. In particular, a long-standing dogma holds that replicative senescence involves resistance to apoptosis, a belief that has led to considerable confusion concerning the role of apoptosis during human ageing. While there are data suggesting that apoptotic cell death plays a key role for ageing in vivo and in the pathogenesis of various age-associated diseases, this is not reflected in the current literature on in vitro senescence. In this article, I summarize key findings concerning the relationship between apoptosis and ageing in vivo and also review the literature concerning the role of apoptosis during in vitro senescence. Recent experimental findings, summarized in this article, suggest that apoptotic cell death (and probably other forms of cell death) are important features of the ageing process that can also be recapitulated in tissue culture systems to some extent. Another important lesson to learn from these studies is that mechanisms of in vitro senescence differ considerably between various histotypes.
\end{abstract}

KEY WORDS: ageing, senescence, apoptosis, fibroblasts, endothelial cell

DOMAINS: genetics (man), molecular biology, cell and tissue culture, cell biology, cell cycle, cell death, aging, atherosclerosis, cardiovascular biology

\section{REPLICATIVE SENESCENCE - A MODEL FOR HUMAN AGEING?}

Human ageing is accompanied by a degeneration of various tissues that lose part of their physiological functions. Tissue degeneration is often accompanied by the loss of specialized cell types. In some cases, this is due to an exhaustion of the cell-division capacity, as is best documented for ageing of the immune system (for review, see [1]), and for part of the skin ageing program (for review, see [2]). At the cellular level, ageing phenomena can be reproduced in vitro 
to some extent for cells derived from various tissues. The most prominent example is human diploid fibroblasts, which display a limited proliferation potential in vitro before they enter a stable growth arrest, defined as replicative senescence (for review, see [3]). Similar results have been described for primary human keratinocytes[4]. Moreover, replicative senescence was also observed in human T lymphocytes[1], human osteoblasts[5], and human endothelial cells[6], although in these cases, details of the senescent phenotype are less clear.

It has been shown that human fibroblasts arrest in the G1 phase of the cell cycle upon entering the senescent state (for review, see [7]), suggesting that deregulation of cell cycle progression may contribute to the senescent phenotype. Progression through the cell cycle is controlled by a family of cyclin-dependent kinases (cdks), heterodimeric enzymes that consist of a catalytic subunit and a specific cyclin that serves as a regulatory subunit[8]. The activity of cdks is controlled through cellular inhibitor proteins, referred to as cdkis (cyclin-dependent kinase inhibitors), which block the catalytic activity of specific cdks through physical association (for review, see [9]). It was shown that the abundance of the cdks p21(WAF1)[10], p16(INK4A)[11,12], and p27(KIP1)[12a] is strongly increased in senescent fibroblasts, which results in the inhibition of cdks[7].

While the initial studies of replicative senescence were focussed on the fact that senescent cells stop to proliferate, more recently there has been a shift of paradigm. There is now increasing evidence that age-associated changes in cellular differentiation and cell physiology contribute significantly to the loss of organ function. In this scenario, the appearance of a few senescent cells in a given tissue is a key determinant of age-related changes of tissue function. This is best illustrated with the example of human skin fibroblasts, which were shown to produce less collagen and to secrete more matrix-degrading enzymes during ageing in vitro (for review, see [2]). This fits well with the observation that the skin of aged individuals is characterized by a decreased collagen content, leading to the gross changes in skin morphology known as wrinkles. Moreover, it was also shown that senescent fibroblasts secrete large amounts of growth factors that support the growth of adjacent preneoplastic cells[13]. In studies of human skin biopsies, an age-related increase of senescent cells was observed in vivo[14], which may account for the agerelated changes in skin morphology as well as the so-far unexplained age-related increases in certain types of skin cancer.

It has been hypothesized that oxidative stress is a major determinant of human ageing in vivo, and there is increasing evidence that this aspect of the ageing process can be reproduced in vitro. Thus, it has been shown that increasing the concentration of reactive oxygen species (ROS), for example, by increasing the oxygen partial pressure[15] or by treating cells with $\mathrm{H}_{2} \mathrm{O}_{2}$ [16], can induce premature senescence in young diploid human fibroblasts. $\mathrm{H}_{2} \mathrm{O}_{2}$-treated human fibroblasts display cell-cycle arrest, senescence-associated $\beta$-galactosidase (SA $\beta$ gal) staining, and other features of spontaneously senescent cells[17,18]. While these data suggest that oxidative stress contributes to the in vitro senescence phenotype of human fibroblasts, the role of reactive oxygen species for the ageing of other human cell types remains to be clarified.

\section{THE ROLE OF PROGRAMMED CELL DEATH IN HUMAN AGEING}

Apoptotic cell death apparently plays an important role during ageing of various tissues in vivo (for review, see [19]), and it was shown recently that mice with a genetic defect in stress-related apoptosis display a significantly induced lifespan[20]. Tissue damage caused by age-dependent apoptosis has been documented in experimental animals for the brain[21], the inner ear[22], and the corpus luteum[23]. Similarly, it has been suggested that age-related apoptosis of $T$ lymphocytes is an important determinant of immunosenescence in humans (for review, see [24]). On the other hand, it is assumed that apoptosis plays an important role in tissue homeostasis, and the failure of cells to exert the apoptotic program can also lead to disorders that accumulate 
during ageing. For example, it was suggested that decreased efficiency of apoptosis contributes to the alterations characteristic of intrinsic (chronologic ageing) and extrinsic (photoageing) skin ageing[25]. Together, these results suggest that regulation of programmed cell death plays an important role for the ageing process in vivo; however, the role of apoptosis for ageing may differ among various tissues.

Programmed cell death (apoptosis) can be triggered by a wide variety of environmental stimuli, and the apoptotic response of a given cell is modified by a plethora of cellular gene products (see box for details). At the cellular level, the issue of age-associated apoptosis is controversial. On the one hand, it was described that human $\mathrm{T}$ cells isolated from elderly persons display increased apoptosis[26], and apoptosis occurs in vitro after prolonged cultivation of CD4+ T cells[27]. On the other hand, the development of apoptosis resistance is also discussed as a key mechanism of immunosenescence[28], and senescent fibroblasts, which display no significant spontaneous apoptosis, are even resistant to proapoptotic signals[29]. Similarly, no increase in the rate of apoptosis was detected during in vitro ageing of human keratinocytes[30]. A recent study further explains the phenomenon of apoptosis resistance in senescent fibroblasts. Seluanov et al.[31] demonstrated that senescent human fibroblasts are indeed resistant to apoptosis, when apoptosis is induced with agents known to activate p53. In that study, the inability of senescent fibroblasts to undergo p53-dependent apoptosis was due to the inability of senescent fibroblasts to activate pathways that lead to p53 stabilization. When senescent fibroblasts are treated with agents that normally would induce p53, such as actinomycinD or UV irradiation, this causes a nonapoptotic type of cell death, namely necrosis[31]. However, p53independent signals are well capable to induce apoptosis in senescent fibroblasts[31].

For ageing of the vascular system, a tissue-damaging role of apoptosis is well established[32]. Arteriosclerosis, a major age-related disease of humans, is accompanied by a degeneration of vascular endothelial cells and vascular smooth muscle cells[33] due to programmed cell death. In this context, the activation of the cellular suicide pathway leading to apoptosis of the endothelial cells represents an initial step in the development of arteriosclerotic lesions[34]. Increased incidence of apoptosis in vitro was also observed when vascular smooth muscle cells from human atherosclerotic plaques were grown in vitro and compared to control cells[35]. Whereas there is compelling evidence that cell death by apoptosis contributes to vascular damage under pathological conditions (e.g., arteriosclerosis), the role of apoptosis during normal vascular ageing remains to be established. Our finding that endothelial cells undergo ageassociated apoptosis during in vitro ageing ([36]; see below) may provide a good model system to study this further.

\section{AGE-ASSOCIATED APOPTOSIS OF ENDOTHELIAL CELLS}

To establish a system for studying vascular ageing at the molecular level, we have grown human umbilical vein endothelial cells (HUVEC) into replicative senescence and compared these cells to the standard fibroblast model of senescence. We found that, unlike senescent fibroblasts that enter a stable G1-arrest phenotype, senescent endothelial cells become polyploid and undergo significant programmed cell death. In senescent cells of both cell types, we found a similar accumulation of G1 cyclins and the cdkis p16, p21, and p27. The data suggest that the mechanisms leading to G1 arrest are probably shared between fibroblasts and endothelial cells; however, there are clearly profound cell type-specific differences in the senescence program with respect to the regulation of ploidy and apoptosis[36]. Interestingly, about $5 \%$ of the cells in the senescent HUVEC population stain positive for cyclin A at a given time point. It was shown that cyclin A gene expression is strictly dependent on activation of cyclin-dependent kinase 2 (cdk2)[37], suggesting that cdk2 remains active in a subpopulation of the senescent HUVEC[36]. Dysregulation of cdk activity was implicated in both apoptosis induction[38] and 
polyploidization[39,40,41]. Hence, the failure of senescent HUVEC cultures to completely downregulate cdks may explain both the proneness to apoptosis and the occurrence of polyploid cells. In a model derived from these findings, the low percentage of S-phase cells in a senescent HUVEC population could give rise to apoptotic cells. Age-related apoptosis is believed to play an important role during tissue ageing[21,22,23], and in vitro senescence of HUVEC may provide a new model for age-related apoptosis that could be useful for studying the role of intrinsic and extrinsic ageing in certain vascular pathologies, e.g., arteriosclerosis, which is characterized by excessive apoptosis[34].

\section{Box 1: Overview of apoptosis regulation in mammalian cells}

While much remains to be learned about apoptotic signal transduction and apoptosis execution, the current knowledge can be briefly summarized as follows. Apoptosis triggered by cell death receptors (e.g., the fas/Fas ligand system[42]) leads to the activation of a class of proteases, referred to as caspases[43], in particular caspase 8 and 10, which activate effector caspases (e.g., caspases 3, 6 or 7)[44]. Effector caspases then cleave key substrates and thereby cause nuclear fragmentation. In an alternative pathway, mitochondrial function is altered through a variety of signals, ultimately leading to the opening of the mitochondrial permeability transition pore (PTP). PTP opening is modulated, among others, by members of the Bcl-2 gene family (for review, see [45]). Once the PTP is open, soluble factors are released from the mitochondrial matrix that trigger, in one way or the other, an apoptotic response. These factors include cytochrome $\mathrm{C}$ which, in combination with the cellular protein Apaf-1[46], leads to activation of caspase 9. Caspase 9 in turn activates effector caspases (see above) and thereby results in cell death[47]. On the other hand, additional factors are released upon PTP opening. There is increasing evidence that mitochondrial death effectors do not necessarily activate caspases, and not all of these factors have been characterized. Recently, apoptosis inducing factor (AIF)[48] and endonuclease G[49] were shown to function as apoptotic effectors that are released from mitochondria and trigger caspase-independent cell death.

\section{REFERENCES}

1. Effros, R.B. (1996) Insights on immunological aging derived from the T-lymphocyte cellular senescence model. Exp. Gerontol. 31(1-2), 21-27.

2. Campisi, J. (1998) The role of cellular senescence in skin aging. J. Invest. Dermatol. Symp. Proc. 3(1), 1-5.

3. Hayflick, L. (1992) Aging, longevity, and immortality in vitro. Exp. Gerontol. 27(4), 363-368.

4. Loughran, O. et al. (1996) Association of CDKN2A/p16INK4A with human head and neck keratinocyte replicative senescence: relationship of dysfunction to immortality and neoplasia. Oncogene 13(3), 561-568.

5. Kassem, M. et al. (1997) Demonstration of cellular aging and senescence in serially passaged longterm cultures of human trabecular osteoblasts. Osteoporos. Int. 7(6), 514-524.

6. Johnson, L.K. and Longenecker, J.P. (1982) Senescence of aortic endothelial cells in vitro: influence of culture conditions and preliminary characterization of the senescent phenotype. Mech. Ageing Dev. 18(1), 118.

7. Stein, G.H. and Dulic, V. (1995) Origins of G(1) arrest in senescent human fibroblasts. Bioessays 17(6), 537-543.

8. Nigg, E.A. (1995) Cyclin-dependent protein kinases: key regulators of the eukaryotic cell cycle. Bioessays 17(6), 471-480.

9. Peter, M. (1997) The regulation of cyclin-dependent kinase inhibitors (CKIs). Prog. Cell. Cycle Res. 3, 99108.

10. Johnson, M. et al. (1994) Evidence for a p53-independent pathway for upregulation of SDI1/CIP1/WAF1/p21 RNA in human cells. Mol. Carcinog. 11(2), 59-64.

11. Alcorta, D.A. et al. (1996) Involvement of the cyclin-dependent kinase inhibitor p16 (INK4a) in replicative senescence of normal human fibroblasts. Proc. Natl. Acad. Sci. U. S. A. 93(24), 13742-13747.

12. Hara, E. et al. (1996) Regulation of p16CDKN2 expression and its implications for cell immortalization and senescence. Mol. Cell. Biol. 16(3), 859-867.

12a. Wagner, M. et al. (2001) Metabolic stabilization of p27 in senescent fibroblasts correlates with reduced expression of the F-box prtein Skp2. Exp. Gerontol. 37, 41-55.

13. Krtolica, A. et al. (2001) Senescent fibroblasts promote epithelial cell growth and tumorigenesis: a link between cancer and aging. Proc. Natl. Acad. Sci. U. S. A. 98(21), 12072-12077. 
14. Dimri, G.P. et al. (1995) A biomarker that identifies senescent human cells in culture and in aging skin in vivo. Proc. Natl. Acad. Sci. U. S. A. 92(20), 9363-9367.

15. von Zglinicki, T. et al. (1995) Mild hyperoxia shortens telomeres and inhibits proliferation of fibroblasts: a model for senescence? Exp. Cell. Res. 220(1), 186-193.

16. Chen, Q. and Ames, B.N. (1994) Senescence-like growth arrest induced by hydrogen peroxide in human diploid fibroblast F65 cells. Proc. Natl. Acad. Sci. U. S. A. 91(10), 4130-4134.

17. Chen, Q.M. et al. (1998) Molecular analysis of H2O2-induced senescent-like growth arrest in normal human fibroblasts: p53 and Rb control G1 arrest but not cell replication. Biochem. J. 332(Pt 1), 43-50.

18. Chen, Q.M., Liu, J., and Merrett, J.B. (2000) Apoptosis or senescence-like growth arrest: influence of cell-cycle position, p53, p21 and bax in $\mathrm{H} 2 \mathrm{O} 2$ response of normal human fibroblasts. Biochem. J. 347(Pt 2), 543-551.

19. Warner, H.R., Hodes, R.J., and Pocinki, K. (1997) What does cell death have to do with aging? J. Am. Geriatr. Soc. 45(9), 1140-1146.

20. Migliaccio, E. et al. (1999) The p66shc adaptor protein controls oxidative stress response and life span in mammals. Nature 402(6759), 309-313.

21. Adams, J.D. et al. (1996) Apoptosis and oxidative stress in the aging brain. Ann. N.Y. Acad. Sci. 786, $135-151$.

22. Usami, S. et al. (1997) Cell death in the inner ear associated with aging is apoptosis? Brain Res. 747(1), 147150.

23. Nicosia, S.V. et al. (1995) Cell proliferation and apoptosis during development and aging of the rabbit corpus luteum. Ann. Clin. Lab. Sci. 25(2), 143-157.

24. Potestio, M. et al. (1998) Apoptosis and ageing. Mech. Ageing Dev. 102(2-3), 221-237.

25. Haake, A.R., Roublevskaia, I., and Cooklis, M. (1998) Apoptosis: a role in skin aging? J. Investig. Dermatol. Symp. Proc. 3(1), 28-35.

26. Aggarwal, S. and Gupta, S. (1998) Increased apoptosis of T cell subsets in aging humans: altered expression of Fas (CD95), Fas ligand, Bcl-2, and Bax. J. Immunol. 160(4), 1627-1637.

27. Grubeck-Loebenstein, B., Lechner, H., and Trieb, K. (1994) Long-term in vitro growth of human T cell clones: can postmitotic 'senescent' cell populations be defined? Int. Arch. Allergy Immunol. 104(3), $232-239$.

28. Spaulding, C., Guo, W., and Effros, R.B. (1999) Resistance to apoptosis in human CD8+ T cells that reach replicative senescence after multiple rounds of antigen-specific proliferation. Exp. Gerontol. 34(5), 633-644.

29. Wang, E. (1995) Senescent human fibroblasts resist programmed cell death, and failure to suppress bcl2 is involved. Cancer Res. 55(11), 2284-2292.

30. Norsgaard, H., Clark, B.F., and Rattan, S.I. (1996) Distinction between differentiation and senescence and the absence of increased apoptosis in human keratinocytes undergoing cellular aging in vitro. Exp. Gerontol. 31(5), 563-570.

31. Seluanov, A. et al. (2001) Change of the death pathway in senescent human fibroblasts in response to DNA damage is caused by an inability to stabilize p53. Mol. Cell. Biol. 21(5), 1552-1564.

32. Heinrich, H. and Holz, J. (1998) Myocardial apoptosis in the overloaded and the aging heart: a critical role of mitochondria? Eur. Cytokine Netw. 9(4), 693-695.

33. Galis, Z.S. et al. (1994) Increased expression of matrix metalloproteinases and matrix degrading activity in vulnerable regions of human atherosclerotic plaques. J. Clin. Invest. 94(6), 2493-2503.

34. Bennett, M.R. (1999) Apoptosis of vascular smooth muscle cells in vascular remodelling and atherosclerotic plaque rupture. Cardiovasc. Res. 41(2), 361-368.

35. Bennett, M.R. et al. (1998) Cooperative interactions between RB and p53 regulate cell proliferation, cell senescence, and apoptosis in human vascular smooth muscle cells from atherosclerotic plaques. Circ. Res. 82(6), 704-712.

36. Wagner, M. et al. (2001) Replicative senescence of human endothelial cells in vitro involves G1 arrest, polyploidization and senescence-associated apoptosis. Exp. Gerontol. 36, 1327-1347.

37. Zerfaß-Thome, K. et al. (1997) $\mathrm{p} 27^{\mathrm{KIP} 1}$ blocks cyclin E-dependent trans-activation of cyclin A gene expression. Mol. Cell. Biol. 17, 407-415.

38. Lipinski, M.M. and Jacks, T. (1999) The retinoblastoma gene family in differentiation and development. Oncogene 18(55), 7873-7882.

39. Stewart, Z.A., Leach, S.D., and Pietenpol, J.A. (1999) p21(Waf1/Cip1) inhibition of cyclin E/Cdk2 activity prevents endoreduplication after mitotic spindle disruption. Mol. Cell. Biol. 19(1), 205-215.

40. Garcia, P. and Cales, C. (1996) Endoreplication in megakaryoblastic cell lines is accompanied by sustained expression of G1/S cyclins and downregulation of cdc25C. Oncogene 13(4), 695-703.

41. Niculescu, A.B. et al. (1998) Effects of p21(Cip1/Wafl) at both the G1/S and the G2/M cell cycle transitions: $\mathrm{pRb}$ is a critical determinant in blocking DNA replication and in preventing endoreduplication. Mol. Cell. Biol. 18(1), 629-643.

42. Walczak, H. and Krammer, P.H. (2000) The CD95 (APO-1/Fas) and the TRAIL (APO-2L) apoptosis systems. Exp. Cell. Res. 256(1), 58-66.

43. Kumar, S. and Colussi, P.A. (1999) Prodomains-adaptors-oligomerization: the pursuit of caspase activation in apoptosis. Trends Biochem. Sci. 24(1), 1-4. 
44. Nicholson, D.W. (1999) Caspase structure, proteolytic substrates, and function during apoptotic cell death. Cell Death Differ. 6(11), 1028-1042.

45. Bernardi, P. et al. (2001) A mitochondrial perspective on cell death. Trends Biochem. Sci. 26(2), 112-117.

46. Zou, H. et al. (1997) Apaf-1, a human protein homologous to C. elegans CED-4, participates in cytochrome c-dependent activation of caspase-3. Cell 90(3), 405-413.

47. Bratton, S.B. et al. (2001) Recruitment, activation and retention of caspases-9 and -3 by Apaf-1 apoptosome and associated XIAP complexes. EMBO J. 20(5), 998-1009.

48. Lorenzo, H.K. et al. (1999) Apoptosis inducing factor (AIF): a phylogenetically old, caspase-independent effector of cell death. Cell Death Differ. 6(6), 516-524.

49. Li, L.Y., Luo, X., and Wang, X. (2001) Endonuclease G is an apoptotic DNase when released from mitochondria. Nature. 412(6842), 95-99.

This article should be referenced as follows:

Jansen-Dürr, P. (2002) Cell death and ageing — a question of cell type. TheScientificWorldJOURNAL 2, 943-948.

\section{Handling Editor:}

Efstathios Gonos, Associate Editor for Aging — a domain of TheScientificWorldJOURNAL.

\section{BIOSKETCH}

Pidder Jansen-Dürr is the head of the Department of Molecular and Cellular Biology of the Institute for Biomedical Ageing Research of the Austrian Academy of Sciences, Innsbruck, Austria. Dr. Pidder JansenDürr has studied Biology and holds a Ph.D. in Zoology. His research interests include cellular ageing, cell cycle control, apoptosis regulation, viral oncogenesis, and metabolic control. 

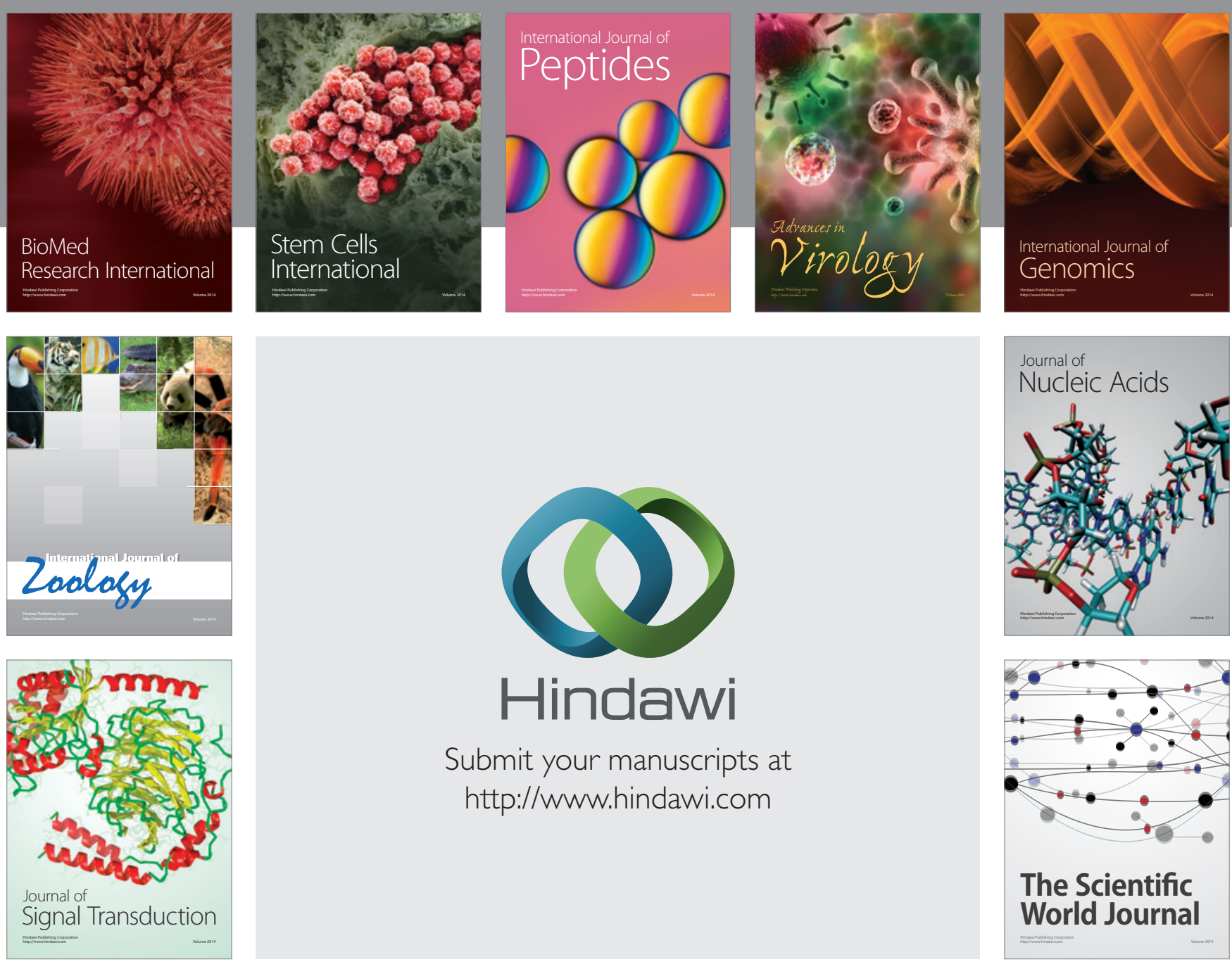

Submit your manuscripts at

http://www.hindawi.com
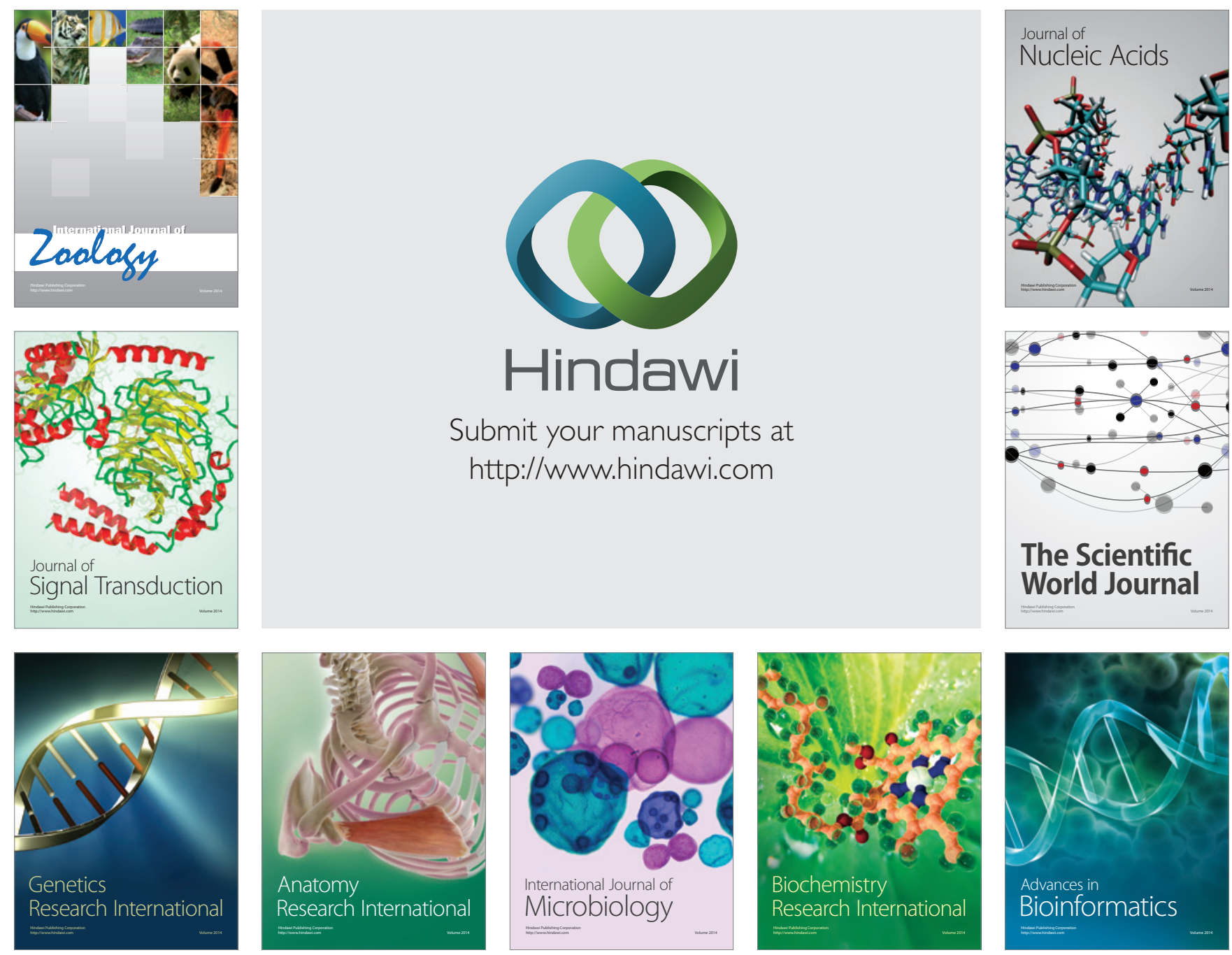

The Scientific World Journal
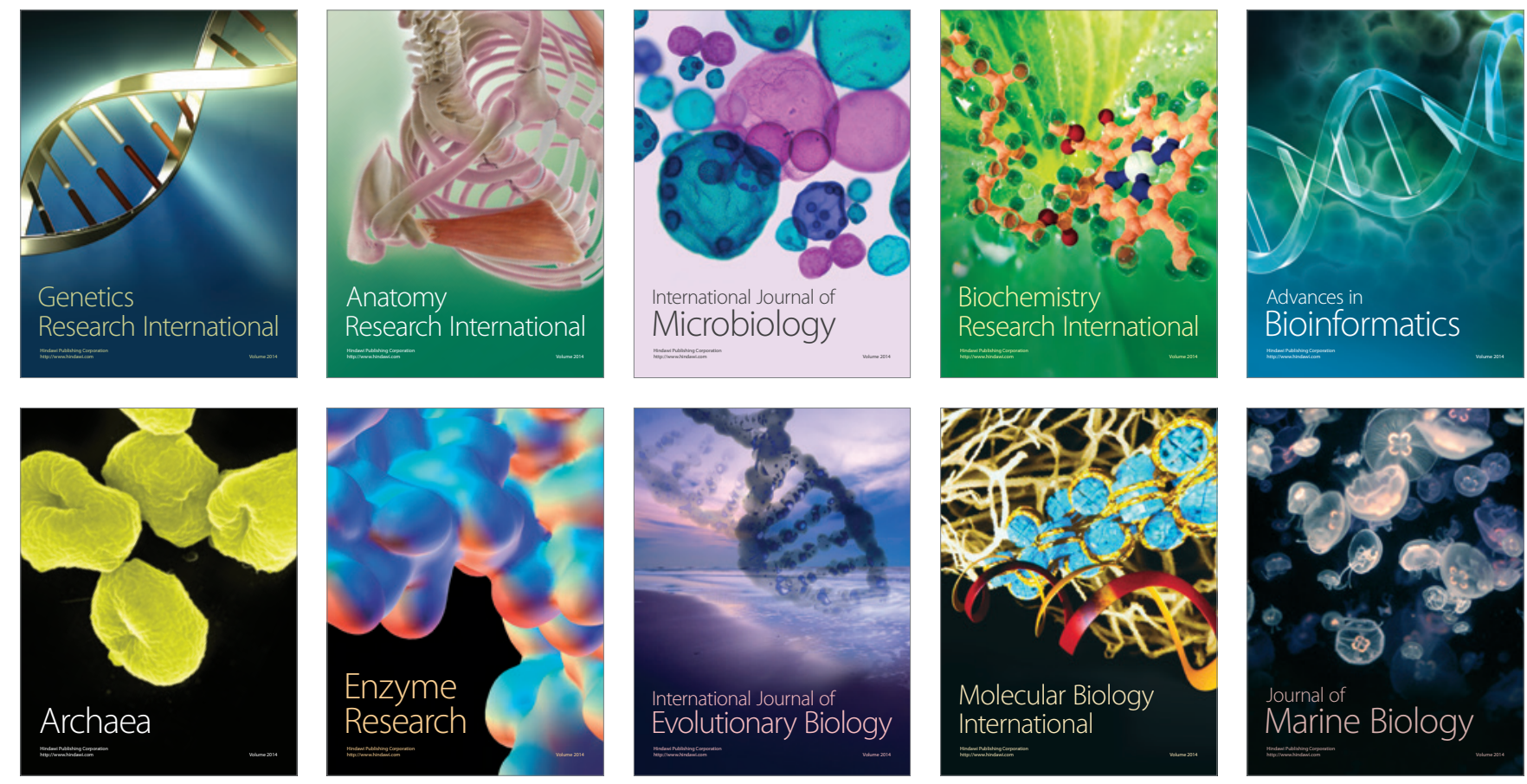